\section{Drug-induced systemic lupus erythematosus: should immune checkpoint inhibitors be added to the evolving list?}

Arnaud and colleagues used WHO's VigiBase, an international spontaneous reporting system, to compile an updated list (19 March 2018) of drugs suspected to be implicated in drug-induced systemic lupus erythematosus (SLE), an evolving clinical entity. ${ }^{1}$ They analysed 12166 reports of drug-induced SLE and identified 118 suspected drugs with pharmacovigilance signal, which were reported in 8163 cases, mainly occurring in women (81\%, 49 years as median age), defined as serious (55\%), with a median onset of 172 days. Of note, 76 drugs (64.4\%) were already known to cause SLE in the literature, including anti-TNF agents (infliximab received the highest number of reports), procainamide and hydralazine (receiving the highest disproportional reporting).

Our attention was drawn to the lack of pharmacovigilance signal for immune checkpoint inhibitors (ICIs), emerging oncological drugs recently associated with a unique and distinct spectrum of side effects, the so-called immune-related adverse events (irAEs), virtually affecting any organ or tissue, with rheumatic manifestations including arthralgia/arthritis, myalgia/myositis, polymyalgia rheumatica, rheumatoid arthritis and Sjögren's syndrome. $^{23}$

Therefore, we analysed the FDA Adverse Event Reporting System (FAERS) to verify whether SLE is reported with ICIs, and characterise relevant cases in terms of severity (eg, hospitalisation), mortality (death reported as outcome), onset time (in relation to ICI regimen), concomitant drugs known to cause SLE and coreported irAEs. ${ }^{4}$ Among 4870 rheumatic events (arthralgia, $\mathrm{n}=711$ ), SLE was reported in 18 cases (as of June 2018), plus 7 cases of cutaneous SLE (two recorded as subacute), 2 cases of lupus-like syndrome and 1 case each for lupus nephritis and central nervous system lupus. Among 18 cases of SLE, only inhibitors of programmed cell death 1 or its ligand (PD1/PDL1) were reported: nivolumab was the suspect ICI in 12 cases, followed by pembrolizumab (4 cases), avelumab and atezolizumab (one each). Mean age was 61 years, with female:male ratio of 1.6; hospitalisation was recorded in four cases, with only one death. The median onset time (calculated for eight cases with available information on event date and start of therapy) was 196 days. Notably, no anti-TNF drugs, procainamide or hydralazine were recorded among concomitant drugs; SLE was the only adverse event recorded in 10 cases, and arthralgia, arthritis and other rheumatic events co-occurred in only 2 cases.

These findings are partially in line with Arnaud and colleagues and open a question on whether ICIs should be added to the list of drug-induced SLE. We hypothesised that ICIs did not emerge with a pharmacovigilance signal from WHO's VigiBase because of potential drug-related and event-related competition bias; that is, the substantial over-reporting of SLE with hydralazine and procainamide, together with the large reporting of irAE with ICIs other than rheumatic events, might have masked the ability to detect disproportionality for events with low reporting rate. ${ }^{5}$ The most intriguing and unexpected finding from FAERS is that SLE with ICIs does not appear to co-occur with other irAEs, especially rheumatic events, with very low fatality rate and delayed onset (more than 6 months). ${ }^{6}$ Although ICI-related SLE appears rare, the increasing uptake of ICIs in clinical practice strengthens the importance of (1) real-time monitoring of pharmacovigilance databases, such as FAERS and WHO's VigiBase; (2) maintaining awareness and long-lasting vigilance by immunologists, rheumatologists and oncologists of this evolving drug-induced clinical entity. The awaited EULAR recommendations, together with accurate reporting of rheumatological irAEs with ICIs, will increase our understanding and relevant confidence of rheumatologists about mechanistic basis, drug-related and patient-related risk factors, as well as optimal management especially in patients with pre-existing autoimmune diseases. ${ }^{7-10}$

\section{Emanuel Raschi, ${ }^{\oplus}$ Ippazio Cosimo Antonazzo, Elisabetta Poluzzi, Fabrizio De Ponti}

Department of Medical and Surgical Sciences, University of Bologna, Bologna, Italy

Correspondence to Dr Emanuel Raschi, Department of Medical and Surgical Sciences, University of Bologna, Bologna 40126, Italy; emanuel.raschi@unibo.it

Contributors ER provided the first draft of the article; all authors were involved in revising it critically for important intellectual content, and all authors approved the final version to be published. Study conception and design: ER, ICA, EP and FDP. Acquisition of data: ICA. Analysis and interpretation of data: ER, ICA, EP and FDP

Funding The authors have not declared a specific grant for this research from any funding agency in the public, commercial or not-for-profit sectors. The authors are supported by institutional research funds (Ricerca Fondamentale Orientata).

Competing interests None declared.

Patient consent for publication Not required.

Provenance and peer review Not commissioned; internally peer reviewed.

(C) Author(s) (or their employer(s)) 2019. No commercial re-use. See rights and permissions. Published by BMJ.

\section{Check for updates}

To cite Raschi E, Antonazzo IC, Poluzzi E, et al. Ann Rheum Dis Epub ahead of print: [please include Day Month Year]. doi:10.1136/annrheumdis-2019-215819

Received 31 May 2019

Accepted 2 June 2019

Ann Rheum Dis 2019;0:1. doi:10.1136/annrheumdis-2019-215819

\section{REFERENCES}

1 Arnaud L, Mertz P, Gavand P-E, et al. Drug-induced systemic lupus: revisiting the everchanging spectrum of the disease using the WHO pharmacovigilance database. Ann Rheum Dis 2019:78:504-8.

2 Cappelli LC, Gutierrez AK, Baer AN, et al. Inflammatory arthritis and sicca syndrome induced by nivolumab and ipilimumab. Ann Rheum Dis 2017;76:43-50.

3 Belkhir R, Burel SL, Dunogeant L, et al. Rheumatoid arthritis and polymyalgia rheumatica occurring after immune checkpoint inhibitor treatment. Ann Rheum Dis 2017;76:1747-50.

4 Raschi E, Mazzarella A, Antonazzo IC, et al. Toxicities with immune checkpoint inhibitors: emerging priorities from Disproportionality analysis of the FDA adverse event Reporting system. Target Oncol 2019;14:205-21.

5 Raschi E, Poluzzi E, Salvo F, et al. Pharmacovigilance of sodium-glucose cotransporter-2 inhibitors: what a clinician should know on disproportionality analysis of spontaneous reporting systems. Nutr Metab Cardiovasc Dis 2018;28:533-42.

6 Wang DY, Salem J-E, Cohen JV, et al. Fatal toxic effects associated with immune checkpoint inhibitors: a systematic review and meta-analysis. JAMA Oncol 2018;4:1721-8.

7 Tocut M, Brenner R, Zandman-Goddard G. Autoimmune phenomena and disease in cancer patients treated with immune checkpoint inhibitors. Autoimmun Rev 2018;17:610-6.

8 Kostine M, Cappelli LC, Calabrese C, et al. Addressing immune-related adverse events of cancer immunotherapy: how prepared are rheumatologists? Ann Rheum Dis 2019;78:860-2.

9 Kostine M, Rouxel L, Barnetche T, et al. Rheumatic disorders associated with immune checkpoint inhibitors in patients with cancer-clinical aspects and relationship with tumour response: a single-centre prospective cohort study. Ann Rheum Dis 2018;77:393-8.

10 Calabrese L, Mariette X. The evolving role of the rheumatologist in the management of immune-related adverse events (irAEs) caused by cancer immunotherapy. Ann Rheum Dis 2018;77:162-4. 